\title{
Mitotane directly interacts with lipid membranes and alters membrane structure and dynamics
}

\section{P. Müller ${ }^{1}$, St. Theisgen ${ }^{2}$, A. Schirbel ${ }^{3}$, S. Sbiera ${ }^{4}$, I. Haralampiev ${ }^{1}$, M. Fassnacht ${ }^{4,5}$, D. Huster ${ }^{2}$, M. Kroiss ${ }^{4,5}$}

1 Department of Biology, Humboldt University, Berlin; ${ }^{2}$ Institute of Medical Physics and Biophysics, University of Leipzig, Leipzig; ${ }^{3}$ Department of Nuclear Medicine, University Hospital Würzburg, Würzburg; ${ }^{4}$ Department of Medicine I, University Hospital Würzburg, Würzburg; ${ }^{5}$ Comprehensive Cancer Center Mainfranken, University of Würzburg, Würzburg;

\section{Background}

Mitotane (1,1-dichloro-2-[o-chlorophenyl]-2-[p-chlorophenyl]ethane DDD; Figure 1), the only drug approved for treatment of adrenocortical carcinoma (ACC), counteracts both tumor growth and tumoral steroid hormone production. Mitotane is lipophilic and we have shown profound impact on cellular lipid metabolism underlying the adrenal specific toxicity (P09-1). This raises the question how mitotane directly interacts with lipids.

\section{Objective}

To investigate the impact of mitotane and its metabolite DDA on lipid membranes using biophysical techniques.<smiles>Clc1ccc([C@H](c2ccccc2Cl)C(Cl)Cl)cc1</smiles>

\section{Methods}

Interaction of Mitotane and DDA was assessed using Nuclear Overhauser Enhancement Spectroscopy-MR.

Large unilamellar vesicles of different composition were labeled with $0.2 \mathrm{mM}$ of various spin-labeled phospholipids (SL-PL). Vesicles were mixed with Mitotane or DDA giving final concentrations of 3.8 $\mathrm{mM}$ lipids and $0.48 \mathrm{mM}$ Mitotane/DDA. $20 \mathrm{mM}$ sodium ascorbate was added and Electron Spin Resonance (ESR) spectra were recorded at $25^{\circ}$ at different time points using a Bruker ECS 106 spectrometer

\section{Results}

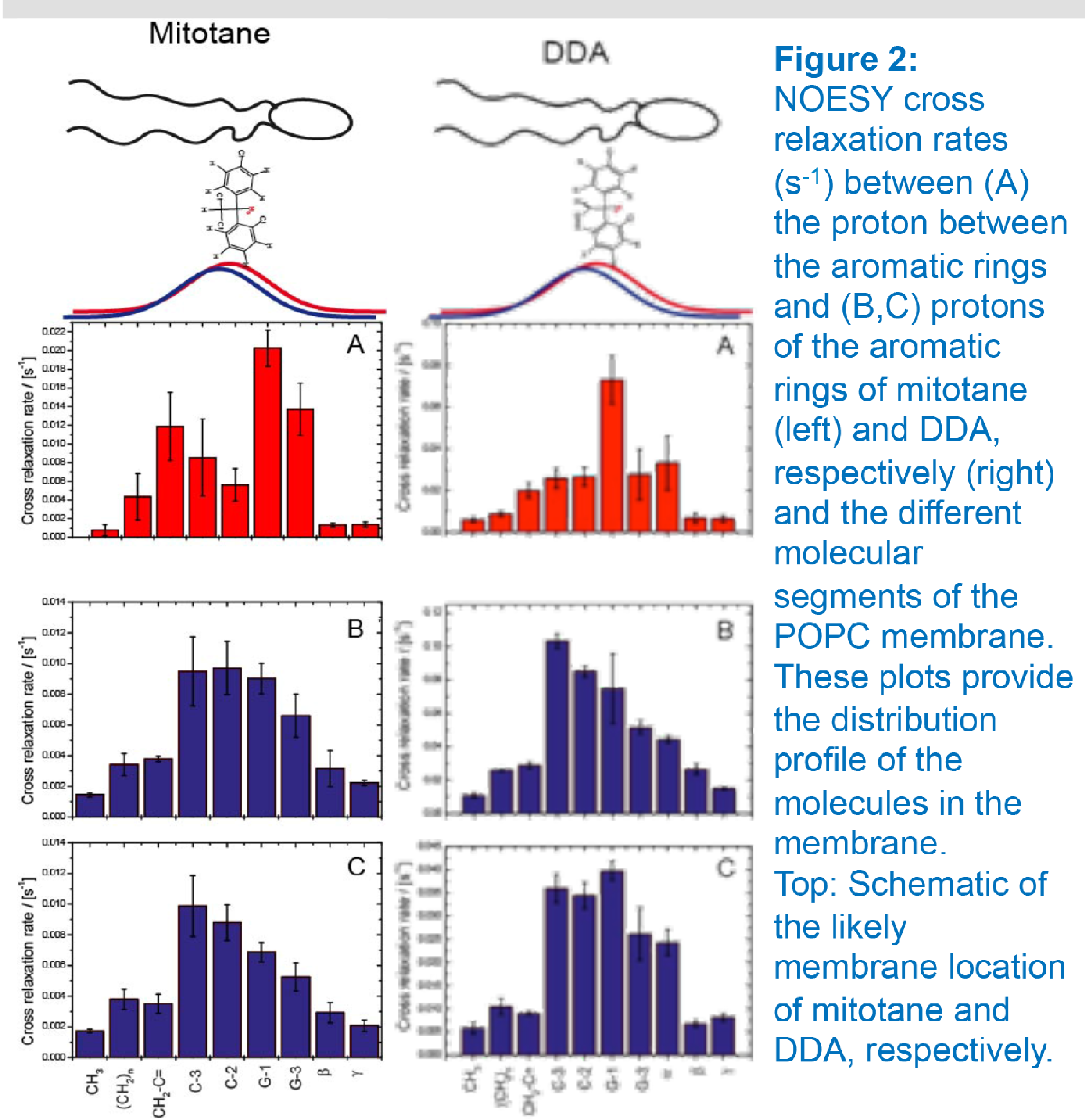

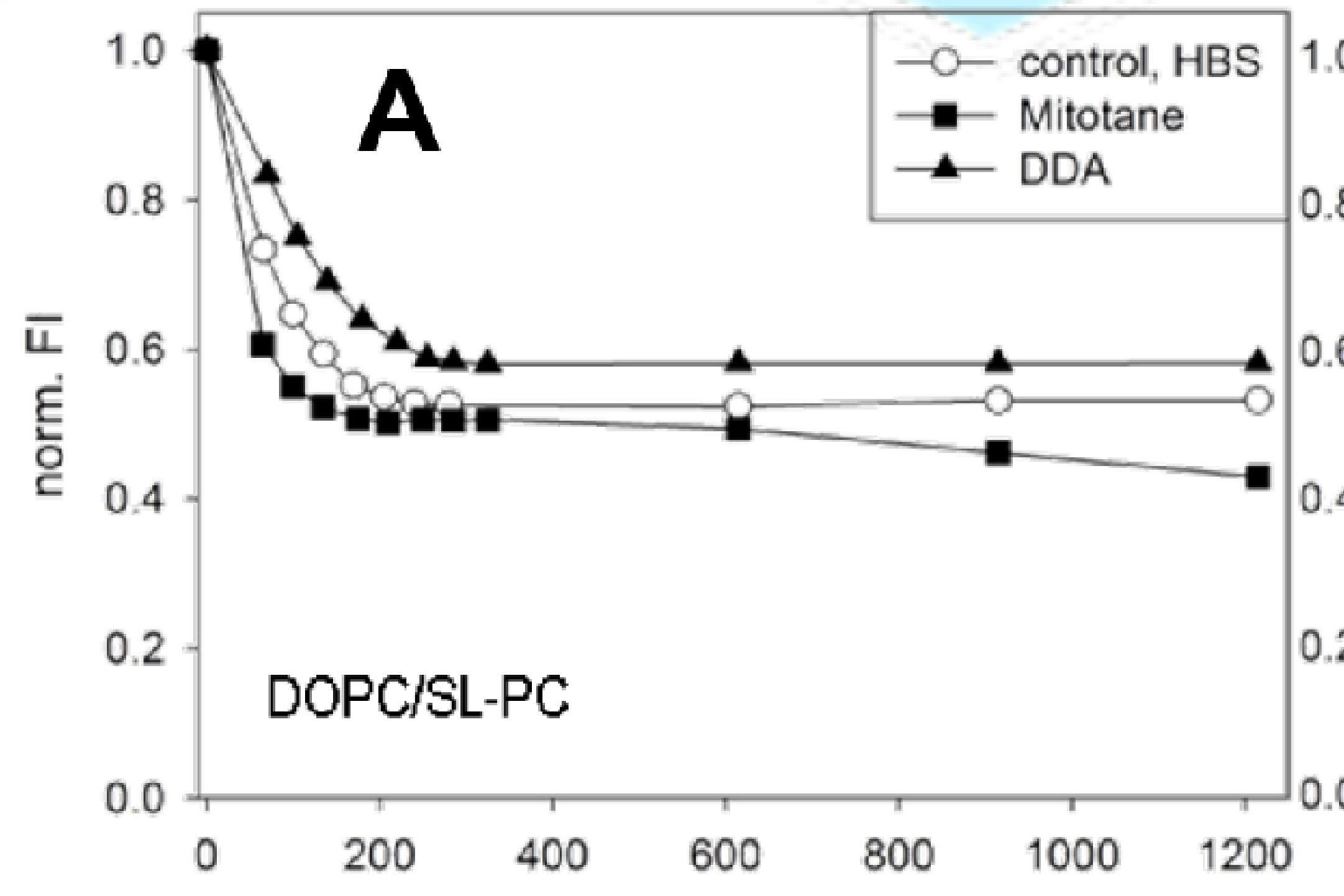
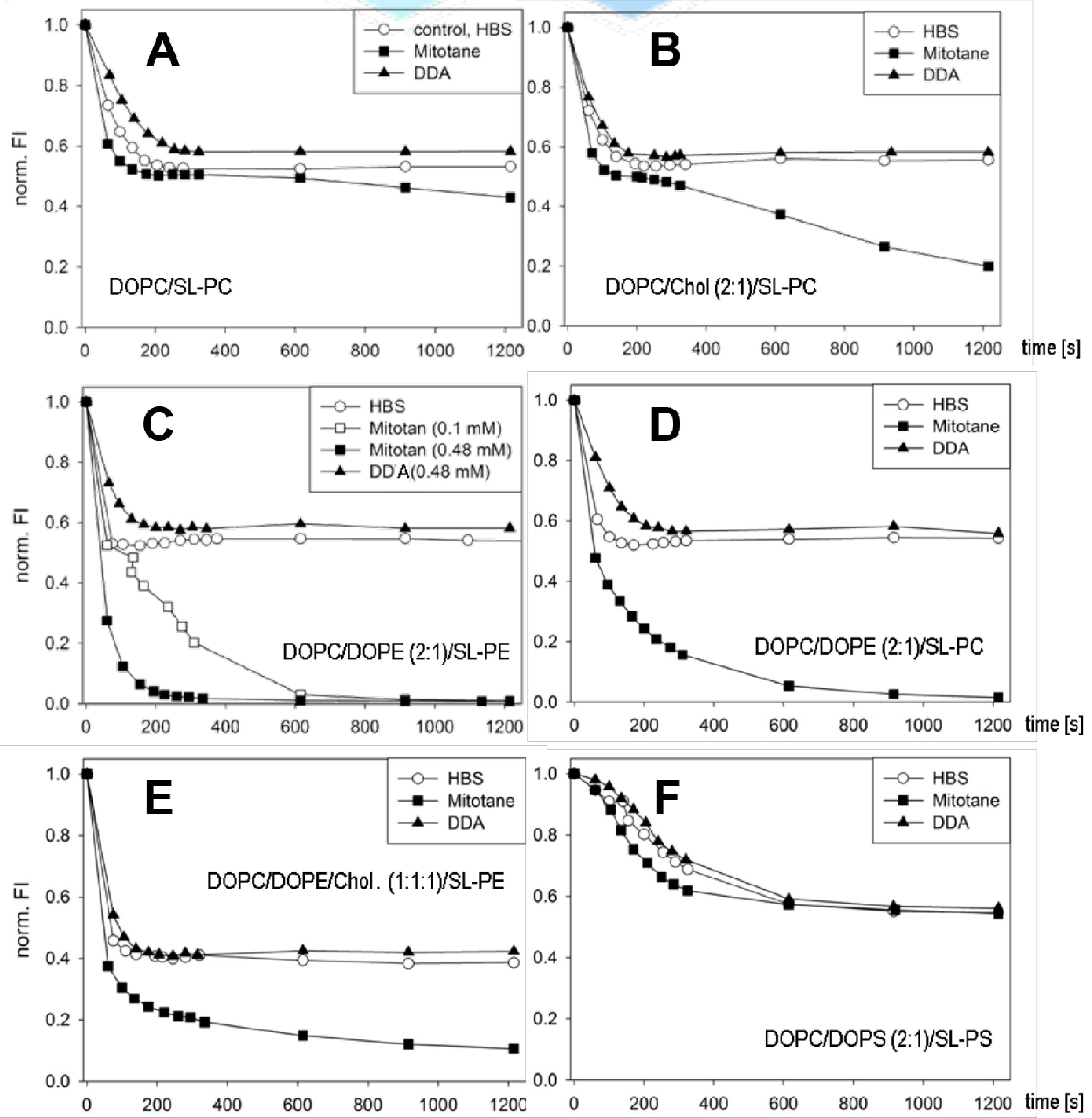

Figure 3: The mitotane metabolite DDA has no influence on membrane integrity at all compositions investigated (A-F). Mitotane has only minor effects on integrity of membranes containing 1,2-dioleoyl-sn-glycero-3-phosphocholine (DOPC) only (A) with a non-significant impact of cholesterol (B). In the presence of phosphatidyl ethanolamine, mitotane causes strong disturbance of membrane integrity as seen by rapid and complete reduction of SL-PE and SL-PC (C, D). This effect is mitotane concentration dependent $(C)$. Presence of cholesterol attenuates this effect $(E)$. Membranes containing phosphatidyl serine remain unaltered $(F)$.

Conclusion:

- mitotane directly interacts with lipid membranes

- intercalation of mitotane into phospholipid bilayers impacts on membrane integrity

- alterations of membrane integrity depend on membrane composition

- mitotane may differentially impact on membrane integrity of organelles

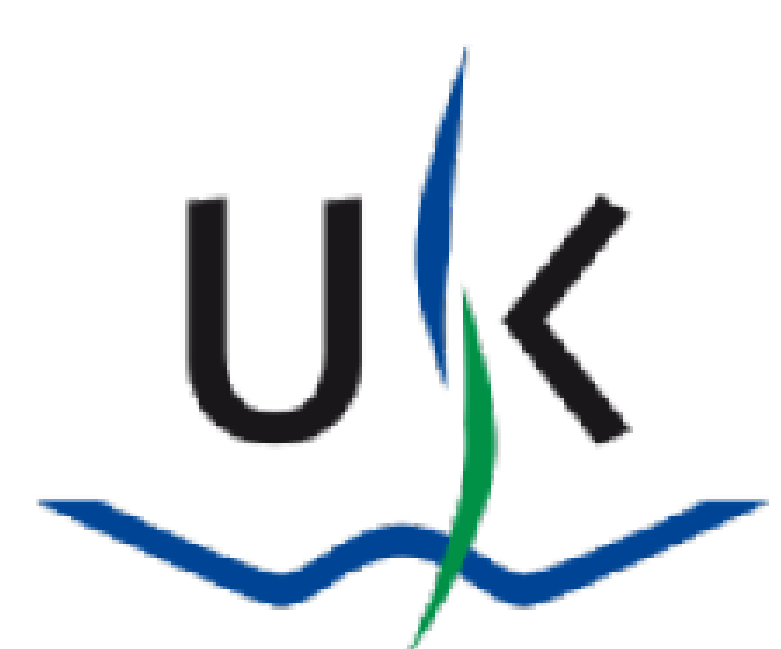

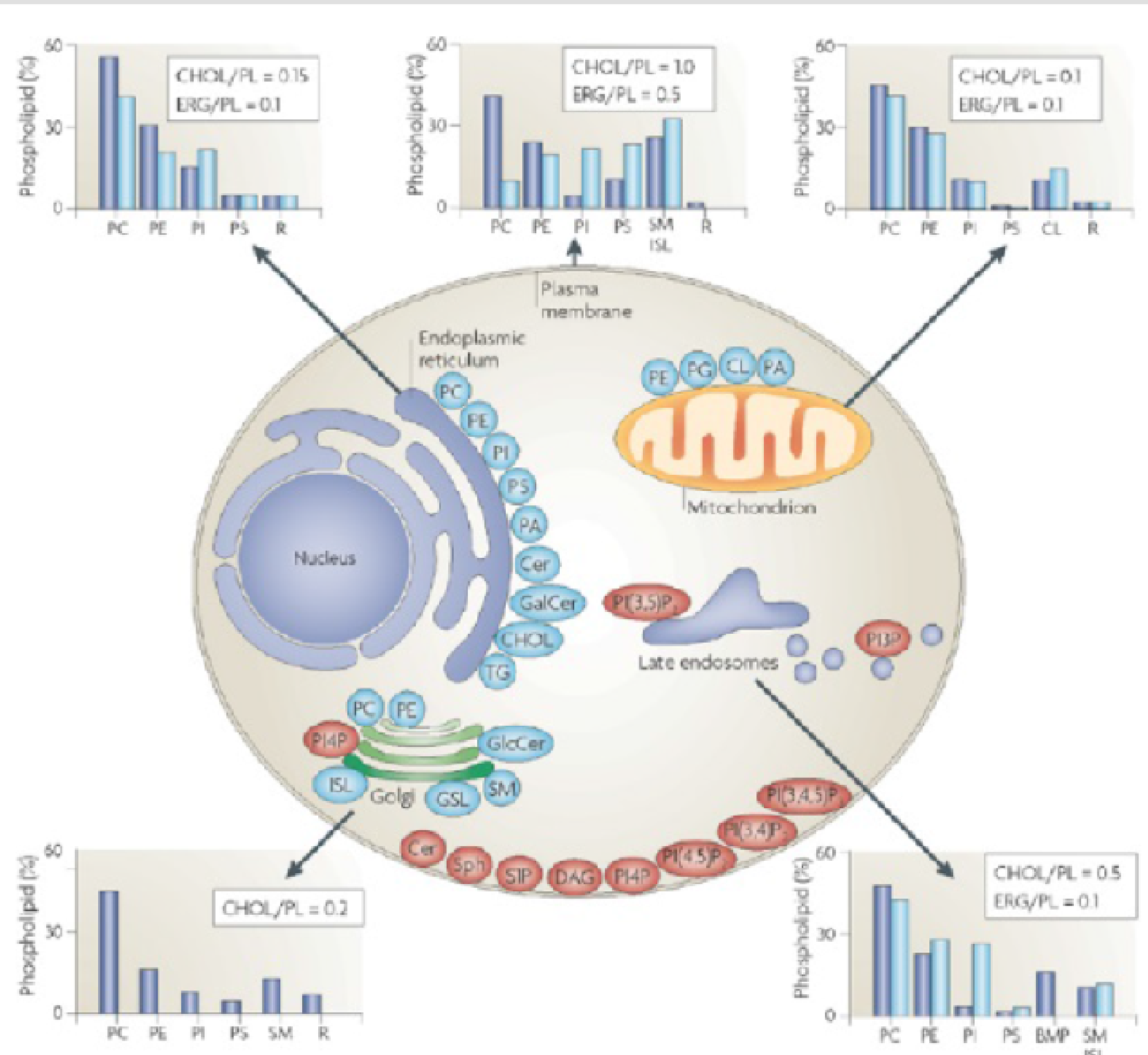

Figure 4: Lipid composition of cell organelles (from van Meer et al., Nature Reviews Molecular Cell Biology 2008)

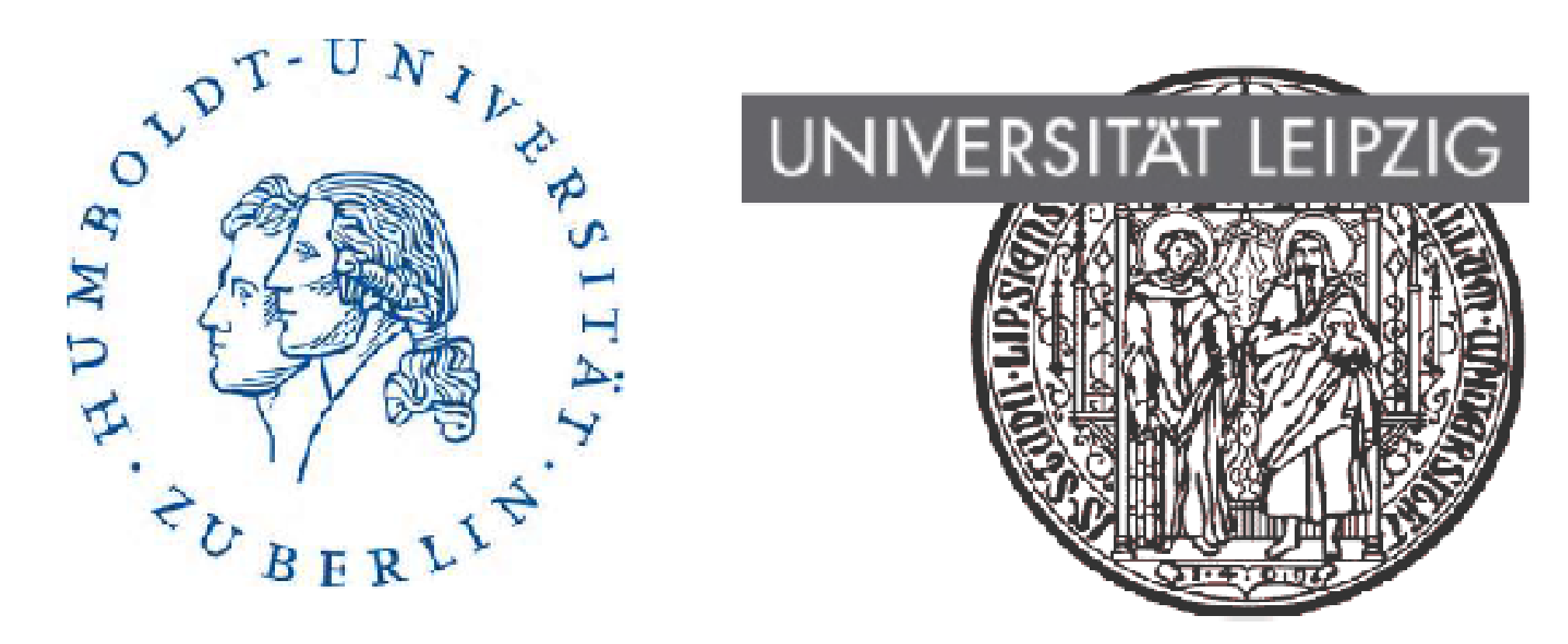

